\title{
On the Cauchy problem for lower semicontinuous differential inclusions
}

\author{
Paolo Cubiotti ${ }^{1}$ and Jen-Chih Yao ${ }^{2,3^{*}}$
}

\section{"Correspondence:} yaojc@mail.cmu.edu.tw

${ }^{2}$ Center for General Education, China Medical University, Taichung, 40402, Taiwan, ROC

${ }^{3}$ Department of Mathematics, King Abdulaziz University, P.O. Box 80203 Jeddah, 21589, Saudi Arabia Full list of author information is available at the end of the article

\section{Springer}

\begin{abstract}
We provide a new proof of a classical result by Bressan on the Cauchy problem for first-order differential inclusions with null initial condition. Our approach allows us to prove the result directly for $k$ th order differential inclusions, under weaker regularity assumptions on the involved multifunction. Our result is the following: let $a, b, M$ be positive real numbers, with $M \cdot \max \left\{a, a^{k}\right\} \leq b$, and let $B$ and $X$ be the closed balls in $\mathbf{R}^{n}$, centered at the origin with radius $b$ and $M$, respectively. Let $F:[0, a] \times B^{k} \rightarrow 2^{X}$ be a multifunction with nonempty closed values, such that $F$ is $\mathcal{L}([0, a]) \otimes \mathcal{B}\left(B^{k}\right)$-measurable, and for all $t \in[0, a]$ the multifunction $F(t, \cdot)$ is lower semicontinuous. Then there exists $u \in W^{k, \infty}\left([0, a], \mathbf{R}^{n}\right)$ such that $u^{(k)}(t) \in F\left(t, u(t), u^{\prime}(t), \ldots, u^{(k-1)}(t)\right)$ a.e. in $[0, a]$, and $u^{(i)}(0)=0_{\mathbf{R}^{n}}$ for all $i=0, \ldots, k-1$.
\end{abstract}

Keywords: differential inclusions; Cauchy problem; generalized solutions; discontinuous selections

\section{Introduction}

The importance of the theory of differential inclusions is well documented in the literature. Indeed, they play a crucial role in the study of many dynamical problems coming from economics, social sciences, biology. They also provide a fundamental tool in control theory. Moreover, differential inclusions are very useful when studying differential equations with discontinuous right-hand side. For a detailed introduction to differential inclusions and their applications, we refer the reader to $[1,2]$.

In the paper [3], A. Bressan proved his classical and celebrated result on the Cauchy problem for first-order differential inclusions, which we now state. In what follows, the space $\mathbf{R}^{n}$ is considered with its Euclidean norm.

Theorem 1.1 (Theorem 2 of [3]) Let $a>0$, and let $B$ and $X$ be the closed balls in $\mathbf{R}^{n}$, centered at the origin with radius $b$ and $M$, respectively, with $a \leq b / M$. Let $F:[0, a] \times B \rightarrow$ $2^{X}$ be a lower semicontinuous multifunction with nonempty closed values.

Then there exists an absolutely continuous $u:[0, a] \rightarrow \mathbf{R}^{n}$ such that $u(0)=0 \mathbf{R}^{n}$ and $u^{\prime}(t) \in F(t, u(t))$ for a.e. $t \in[0, a]$.

The aim of this note is simply to propose an alternative proof of Theorem 1.1, completely independent from the original one. Our approach also allows one to state Theorem $1.1 \mathrm{di}$ rectly for $k$ th-order differential inclusions, under slightly weaker regularity assumptions

(c) 2016 Cubiotti and Yao. This article is distributed under the terms of the Creative Commons Attribution 4.0 International License (http://creativecommons.org/licenses/by/4.0/), which permits unrestricted use, distribution, and reproduction in any medium, provided you give appropriate credit to the original author(s) and the source, provide a link to the Creative Commons license, and indicate if changes were made. 
on $F$. That is, the joint lower semicontinuity of the multifunction $F$ is replaced by joint measurability and by the lower semicontinuity of $F(t, \cdot)$. In this connection, we observe that the original proof of Theorem 1.1 does not work if $F$ is not jointly lower semicontinuous (see the Remark at p.96 of [3]).

Before stating our result, we fix some notations. If $a>0, k, n \in \mathbf{N}$ and $p \in[1,+\infty]$, we denote by $W^{k, p}\left([0, a], \mathbf{R}^{n}\right)$ the set of all $u \in C^{k-1}\left([0, a], \mathbf{R}^{n}\right)$ such that $u^{(k-1)}$ is absolutely continuous in $[0, a]$ and $u^{(k)} \in L^{p}\left([0, a], \mathbf{R}^{n}\right)$. Moreover, we denote by $\mathcal{L}([0, a])$ and $\mathcal{B}(S)$ the family of all measurable subsets of $[0, a]$ and the Borel family of the topological space $S$, respectively. The following is our result.

Theorem 1.2 Let $a, b, M$ be three positive real numbers, with $M \cdot \max \left\{a, a^{k}\right\} \leq b$. Let $X$ and $B$ be as in Theorem 1.1, and let $F:[0, a] \times B^{k} \rightarrow 2^{X}$ be a multifunction with nonempty closed values. Assume that $F$ is $\mathcal{L}([0, a]) \otimes \mathcal{B}\left(B^{k}\right)$-measurable, and that for all $t \in[0, a]$ the multifunction $F(t, \cdot)$ is lower semicontinuous. Then there exists a function $u \in W^{k, \infty}\left([0, a], \mathbf{R}^{n}\right)$ such that

$$
\begin{cases}u^{(k)}(t) \in F\left(t, u(t), u^{\prime}(t), \ldots, u^{(k-1)}(t)\right) & \text { for a.e. } t \in[0, a], \\ u^{(i)}(0)=0_{\mathbf{R}^{n}} & \text { for all } i=0, \ldots, k-1 .\end{cases}
$$

The proof of Theorem 1.2, which will be given in Section 3, will follow from a more general result, Theorem 3.1 below. It is mainly based on a recent existence result for discontinuous selections and on an existence result for operator inclusions, stated below as Theorems 2.1 and 2.3, respectively. In Section 2, conversely, we shall give some notations and recall the results that we shall use in our proofs.

\section{Preliminaries}

Let $n \in \mathbf{N}$. For all $i \in\{1, \ldots, n\}$, we shall denote by $P_{n, i}: \mathbf{R}^{n} \rightarrow \mathbf{R}$ the projection over the $i$ th axis. The space $\mathbf{R}^{n}$ is considered with its Euclidean norm $\|\cdot\|_{n}$. If $A \subseteq \mathbf{R}^{n}$, we denote by $\overline{\operatorname{conv}}(A)$ the closed convex hull of $A$. Moreover, we denote by $m_{n}$ the $n$-dimensional Lebesgue measure in $\mathbf{R}^{n}$. If $[a, b]$ is a compact interval, we shall denote by $A C\left([a, b], \mathbf{R}^{n}\right)$ the space of all absolutely continuos functions from $[a, b]$ into $\mathbf{R}^{n}$.

Let $p \in[1,+\infty]$. The space $L^{p}\left([a, b], \mathbf{R}^{n}\right)$ is considered with the usual norm

$$
\|u\|_{L^{p}\left([a, b], \mathbf{R}^{n}\right)}:= \begin{cases}\left(\int_{a}^{b}\|u(t)\|_{n}^{p} d t\right)^{\frac{1}{p}} & \text { if } p<+\infty \\ \operatorname{essup}_{t \in[a, b]}\|u(t)\|_{n} & \text { if } p=+\infty\end{cases}
$$

As usual, we put $L^{p}([a, b]):=L^{p}([a, b], \mathbf{R})$. Moreover, if $x \in \mathbf{R}^{n}$ and $r>0$, we put

$$
\bar{B}_{n}(x, r):=\left\{v \in \mathbf{R}^{n}:\|v-x\|_{n} \leq r\right\} .
$$

Let $S, V$ be topological spaces, let $F: S \rightarrow 2^{V}$ be a multifunction, and let $x_{0} \in S$. We recall that the multifunction $F$ is said to be lower semicontinuous at $x_{0}$ if for any open set $A \subseteq V$, with $F\left(x_{0}\right) \cap A \neq \emptyset$, there exists an open set $B \subseteq S$ such that

$$
x_{0} \in B \subseteq F^{-}(A):=\{x \in S: F(x) \cap A \neq \emptyset\} .
$$


The multifunction $F$ is said to be lower semicontinuous in $S$ if it is lower semicontinuous at each point $x \in S$. When the multifunction $F$ is single valued, the above notion of lower semicontinuity reduces to the usual notion of continuity (for single-valued maps).

If $(T, \mathcal{A})$ is a measurable space, a multifuncion $F: T \rightarrow 2^{V}$ is said to be measurable (resp., weakly measurable) if for any closed (resp., open) set $W \subseteq V$ one has $F^{-}(W) \in \mathcal{A}$. For the basic definitions and facts about multifunctions, we refer the reader to [4] and [5]. For what concerns measurable multifunctions, we also refer to [6]. In the following, we shall make the obvious identification $\left(\mathbf{R}^{n}\right)^{k}=\mathbf{R}^{n k}$ whenever it is convenient for simpler exposition.

For the sake of completeness, we now recall the results that we use in our proofs. We start with the following very recent selection theorem.

Theorem 2.1 (Theorem 2.2 of [7]) Let $T$ and $X_{1}, X_{2}, \ldots, X_{k}$ be Polish spaces, with $k \in \mathbf{N}$, and let $X:=\prod_{j=1}^{k} X_{j}$ (endowed with the product topology). Let $\mu, \psi_{1}, \ldots, \psi_{k}$ be positive regular Borel measures over $T, X_{1}, X_{2}, \ldots, X_{k}$, respectively, with $\mu$ finite and $\psi_{1}, \ldots, \psi_{k} \sigma$-finite.

Let $S$ be a separable metric space, and let $F: T \times X \rightarrow 2^{S}$ be a multifunction with nonempty complete values. Finally, let $E \subseteq X$ be a given set. For all $t \in T$, let $F_{t}: X \rightarrow 2^{S}$ be the multifunction defined by putting

$$
F_{t}\left(x_{1}, \ldots, x_{k}\right):=F\left(t, x_{1}, \ldots, x_{k}\right) \text {, }
$$

and let $P_{*, i}: X \rightarrow X_{i}$ be the projection over $X_{i}$. Assume that:

(i) the multifunction $F$ is $\mathcal{T}_{\mu} \otimes \mathcal{B}(X)$-measurable $\left(\mathcal{T}_{\mu}\right.$ denoting the completion of the Borel $\sigma$-algebra $\mathcal{B}(T)$ of $T$ with respect to the measure $\mu$ );

(ii) for a.e. $t \in T$, one has

$$
\left\{x:=\left(x_{1}, \ldots, x_{k}\right) \in X: F_{t} \text { is not lower semicontinuous at } x\right\} \subseteq E .
$$

Then there exist sets $Q_{1}, \ldots, Q_{k}$, with

$$
Q_{i} \in \mathcal{B}\left(X_{i}\right) \quad \text { and } \quad \psi_{i}\left(Q_{i}\right)=0 \text { for all } i=1, \ldots, k \text {, }
$$

and a function $\phi: T \times X \rightarrow S$ such that:

(a) $\phi(t, x) \in F(t, x)$ for all $(t, x) \in T \times X$;

(b) for all $x:=\left(x_{1}, x_{2}, \ldots, x_{k}\right) \in X \backslash\left[\left(\bigcup_{i=1}^{k} P_{*, i}^{-1}\left(Q_{i}\right)\right) \cup E\right]$, the function $\phi(\cdot, x)$ is $\mathcal{T}_{\mu}$-measurable over $T$

(c) for a.e. $t \in T$, one has

$$
\begin{aligned}
& \left\{x:=\left(x_{1}, x_{2}, \ldots, x_{k}\right) \in X: \phi(t, \cdot) \text { is discontinuous at } x\right\} \\
& \quad \subseteq E \cup\left[\left(\bigcup_{i=1}^{k} P_{*, i}^{-1}\left(Q_{i}\right)\right)\right] .
\end{aligned}
$$

We also recall the following proposition.

Proposition 2.2 (Proposition 2.6 of [8]) Let $\psi:[a, b] \times \mathbf{R}^{n} \rightarrow \mathbf{R}^{k}$ be a given function, $E \subseteq \mathbf{R}^{n}$ be a Lebesgue measurable set, with $m_{n}(E)=0$, and let $D$ be a countable dense subset of $\mathbf{R}^{n}$, with $D \cap E=\emptyset$. Assume that: 
(i) for all $t \in[a, b]$, the function $\psi(t, \cdot)$ is bounded;

(ii) for all $x \in D$, the function $\psi(\cdot, x)$ is $\mathcal{L}([a, b])$-measurable.

Let $G:[a, b] \times \mathbf{R}^{n} \rightarrow 2^{\mathbf{R}^{k}}$ be the multifunction defined by setting, for each $(t, x) \in[a, b] \times$ $\mathbf{R}^{n}$,

$$
G(t, x)=\bigcap_{m \in \mathbf{N}} \overline{\operatorname{conv}} \overline{\left(\bigcup_{\substack{y \in D \\\|y-x\|_{n} \leq \frac{1}{m}}}\{\psi(t, y)\}\right) .}
$$

Then one has:

(a) G has nonempty closed convex values;

(b) for all $x \in \mathbf{R}^{n}$, the multifunction $G(\cdot, x)$ is $\mathcal{L}([a, b])$-measurable;

(c) for all $t \in[a, b]$, the multifunction $G(t, \cdot)$ has closed graph;

(d) if $t \in[a, b]$, and $\left.\psi(t, \cdot)\right|_{\mathbf{R}^{n} \backslash E}$ is continuous at $x \in \mathbf{R}^{n} \backslash E$, then one has

$$
G(t, x)=\{\psi(t, x)\} .
$$

The following existence result for operator inclusions is due to Naselli Ricceri and Ricceri.

Theorem 2.3 (Theorem 1 of [9]) Let $(T, \mathcal{F}, \mu)$ be a finite non-atomic complete measure space; $V$ a nonempty set; $\left(X,\|\cdot\|_{X}\right),\left(Y,\|\cdot\|_{Y}\right)$ two separable real Banach spaces, with $Y$ finite-dimensional; $p, q, s \in[1,+\infty]$, with $q<+\infty$ and $q \leq p \leq s ; \Psi: V \rightarrow L^{s}(T, Y) a$ surjective and one-to-one operator; $\Phi: V \rightarrow L^{1}(T, X)$ an operator such that, for every $v \in$ $L^{s}(T, Y)$ and every sequence $\left\{v_{n}\right\}$ in $L^{s}(T, Y)$ weakly converging to $v$ in $L^{q}(T, Y)$, the sequence $\left\{\Phi\left(\Psi^{-1}\left(v_{n}\right)\right)\right\}$ converges strongly to $\Phi\left(\Psi^{-1}(v)\right)$ in $L^{1}(T, X) ; \varphi:[0,+\infty[\rightarrow[0,+\infty]$ a nondecreasing function such that

$$
\underset{t \in T}{\operatorname{esssup}}\|\Phi(u)(t)\|_{X} \leq \varphi\left(\|\Psi(u)\|_{L^{p}(T, Y)}\right)
$$

for all $u \in V$.

Further, let $F: T \times X \rightarrow 2^{Y}$ be a multifunction, with nonempty closed convex values, satisfying the following conditions:

(i) for $\mu$-almost every $t \in T$, the multifunction $F(t, \cdot)$ has closed graph;

(ii) the set

$$
\{x \in X: \text { the multifunction } F(\cdot, x) \text { is weakly measurable }\}
$$

is dense in $X$;

(iii) there exists a number $r>0$ such that the function

$$
t \rightarrow \sup _{\|x\|_{X} \leq \varphi(r)} d\left(0_{Y}, F(t, x)\right)
$$

belongs to $L^{s}(T)$ and its norm in $L^{p}(T)$ is less or equal to $r$.

Under such hypotheses, there exists $\tilde{u} \in V$ such that

$$
\begin{aligned}
& \Psi(\tilde{u})(t) \in F(t, \Phi(\tilde{u})(t)) \quad \mu \text {-a.e., } \\
& \|\Psi(\tilde{u})(t)\|_{Y} \leq \sup _{\|x\|_{X} \leq \varphi(r)} d\left(0_{Y}, F(t, x)\right) \quad \mu \text {-a.e. in } T .
\end{aligned}
$$


The two following results, concerning absolutely continuous functions, will be fundamental in the sequel. We recall that a function $f:[a, b] \rightarrow[\alpha, \beta]$ is called an $N$-function if it maps null sets into null sets.

Theorem 2.4 (Theorem 2 of [10]) Let $f:[a, b] \rightarrow \mathbf{R}$ be continuous and strictly monotonic. Then $f^{-1}$ is absolutely continuous if and only if $f^{\prime} \neq 0$ almost everywhere on $[a, b]$.

Theorem 2.5 (Theorem 18.25 of [11]) Let $f$ be a continuous function of finite variation with domain $[a, b] \subseteq \mathbf{R}$ and range $[\alpha, \beta] \subseteq \mathbf{R}$. Then $f$ is an $N$-function if and only iff is absolutely continuous.

Finally, we recall the following lemma.

Lemma 2.6 (Lemma 3.2 of [12]) Let $(T, \mathcal{D}, \mu)$ be a complete finite measure space, $X$ be a Polish space, $Y, Z$ be two topological spaces, $F: T \times X \rightarrow 2^{Z}$ and $H: T \times Y \rightarrow 2^{X}$ be two multifunctions. Assume that:

(a) $F$ is $\mathcal{D} \otimes \mathcal{B}(X)$-measurable;

(b) $H$ is $\mathcal{D} \otimes \mathcal{B}(Y)$-measurable and has closed values.

Then the multifunction $G$ defined by $G(t, y)=F(t, H(t, y))$ for all $(t, y) \in T \times Y$ is $\mathcal{D} \otimes$ $\mathcal{B}(Y)$-measurable.

\section{The result}

Theorem 1.2 above follows from the following more general result.

Theorem 3.1 Let $n, k \in \mathbf{N}, a>0, F:[0, a] \times\left(\mathbf{R}^{n}\right)^{k} \rightarrow 2^{\mathbf{R}^{n}}$ a bounded multifunction with nonempty closed values. Assume that $F$ is $\mathcal{L}([0, a]) \otimes \mathcal{B}\left(\left(\mathbf{R}^{n}\right)^{k}\right)$-measurable, and that for all $t \in[0, a]$ the multifunction $F(t, \cdot)$ is lower semicontinuous. Then there exists a solution $u \in W^{k, \infty}\left([0, a], \mathbf{R}^{n}\right)$ of problem (1).

Proof Let $H:=\overline{\operatorname{conv}}\left(F\left([0, a] \times\left(\mathbf{R}^{n}\right)^{k}\right)\right)$. We now divide the proof into two steps.

Step 1. We first of all assume, in addition, that $H \subseteq \mathbf{R}_{+}^{n}$ (the positive open orthant of $\left.\mathbf{R}^{n}\right)$. Let $M>0$ be such that $H \subseteq \bar{B}_{n}\left(0_{\mathbf{R}^{n}}, M\right)$. By Theorem 2.1, there exist sets $C \in \mathcal{B}(\mathbf{R})$ and $K_{0} \in \mathcal{L}([0, a])$, with $m_{1}(C)=m_{1}\left(K_{0}\right)=0$, and a function $\psi:[0, a] \times\left(\mathbf{R}^{n}\right)^{k} \rightarrow 2^{H}$, such that:

(a) $\psi(t, \xi) \in F(t, \xi)$ for all $(t, \xi) \in[0, a] \times\left(\mathbf{R}^{n}\right)^{k}$;

(b) for all $\xi \in\left(\mathbf{R}^{n}\right)^{k} \backslash\left[\bigcup_{i=1}^{n k} P_{n k, i}^{-1}(C)\right]$, the function $\psi(\cdot, \xi)$ is $\mathcal{L}([0, a])$-measurable;

(c) for all $t \in[0, a] \backslash K_{0}$, one has

$$
\left\{\xi \in\left(\mathbf{R}^{n}\right)^{k}: \psi(t, \cdot) \text { is discontinuous at } \xi\right\} \subseteq\left[\bigcup_{i=1}^{n k} P_{n k, i}^{-1}(C)\right]
$$

Since $m_{n k}\left(\bigcup_{i=1}^{n k} P_{n k, i}^{-1}(C)\right)=0$, there exists a countable set $D \subseteq\left(\mathbf{R}^{n}\right)^{k} \backslash\left[\bigcup_{i=1}^{n k} P_{n k, i}^{-1}(C)\right]$, which is dense in $\mathbf{R}^{n k}$. 
Let $G:[0, a] \times\left(\mathbf{R}^{n}\right)^{k} \rightarrow 2^{\mathbf{R}^{n}}$ be the multifunction defined by setting, for all $(t, \xi) \in[0, a] \times$ $\left(\mathbf{R}^{n}\right)^{k}$,

$$
G(t, \xi):=\bigcap_{m \in \mathbf{N}} \overline{\operatorname{conv}} \overline{\left(\bigcup_{\substack{\eta \in D \\\|\eta-\xi\|_{n k} \leq \frac{1}{m}}}\{\psi(t, \eta)\}\right) .}
$$

By Proposition 2.2 we get:

(a)' $G$ has nonempty closed convex values;

(b)' for all $\xi \in\left(\mathbf{R}^{n}\right)^{k}$, the multifunction $G(\cdot, \xi)$ is $\mathcal{L}([0, a])$-measurable;

(c)' for all $t \in[0, a]$, the multifunction $G(t, \cdot)$ has closed graph;

(d) $)^{\prime}$ if $t \in[0, a]$, and the function $\psi(t, \cdot)$ is continuous at $\xi \in\left(\mathbf{R}^{n}\right)^{k}$, then one has $G(t, \xi)=$ $\{\psi(t, \xi)\}$.

Moreover, we have $G\left([0, a] \times\left(\mathbf{R}^{n}\right)^{k}\right) \subseteq H \subseteq \bar{B}_{n}(0, M)$.

Now, let $\phi_{1}: L^{\infty}\left([0, a], \mathbf{R}^{n}\right) \rightarrow A C\left([0, a], \mathbf{R}^{n}\right)$ be defined by putting, for all $v \in L^{\infty}([0, a]$, $\left.\mathbf{R}^{n}\right)$,

$$
\phi_{1}(v)(t)=\int_{0}^{t} v(s) d s \quad \text { for all } t \in[0, a]
$$

For each $j=2, \ldots, k$, let $\phi_{j}: L^{\infty}\left([0, a], \mathbf{R}^{n}\right) \rightarrow C^{j-1}\left([0, a], \mathbf{R}^{n}\right)$ be defined by putting, for all $v \in L^{\infty}\left([0, a], \mathbf{R}^{n}\right)$,

$$
\phi_{j}(v)(t)=\int_{0}^{t} \phi_{j-1}(v)(s) d s \quad \text { for all } t \in[0, a]
$$

Let $\Phi: L^{\infty}\left([0, a], \mathbf{R}^{n}\right) \rightarrow A C\left([0, a], \mathbf{R}^{n k}\right)$ be defined by putting, for all $v \in L^{\infty}\left([0, a], \mathbf{R}^{n}\right)$,

$$
\Phi(v)(t)=\left(\phi_{k}(v)(t), \phi_{k-1}(v)(t), \ldots, \phi_{1}(v)(t)\right)
$$

for all $t \in[0, a]$. Now we want to apply Theorem 2.3, with $T=[0, a], s=p=+\infty, q=1$, $X=\mathbf{R}^{n k}, Y=\mathbf{R}^{n}, V=L^{\infty}\left([0, a], \mathbf{R}^{n}\right), \Psi(v)=v, \varphi \equiv+\infty, r=M, F=G$ and $\Phi$ defined as above. To this aim, observe what follows.

(a)" For every $v \in L^{\infty}\left([0, a], \mathbf{R}^{n}\right)$, and every sequence $\left\{v_{m}\right\}$ in $L^{\infty}\left([0, a], \mathbf{R}^{n}\right)$, weakly converging to $v$ in $L^{1}\left([0, a], \mathbf{R}^{n}\right)$, the sequence $\left\{\Phi\left(v_{m}\right)\right\}$ converges strongly to $\Phi(v)$ in $L^{1}\left([0, a], \mathbf{R}^{n k}\right)$. To see this, let $\left\{v_{m}\right\}$ and $v$ in $L^{\infty}\left([0, a], \mathbf{R}^{n}\right)$ be fixed, with $\left\{v_{m}\right\}$ weakly convergent to $v$ in $L^{1}\left([0, a], \mathbf{R}^{n}\right)$. It is quite easy to check that the sequence $\left\{\phi_{1}\left(v_{m}\right)\right\}$ converges pointwise in $[0, a]$ to $\phi_{1}(v)$. That is, for every $t \in[a, b]$ one has $\lim _{m \rightarrow \infty}\left\|\phi_{1}\left(v_{m}\right)(t)-\phi_{1}(v)(t)\right\|_{n}=0$. Since $\left\{v_{m}\right\}$ is weakly convergent in $L^{1}\left([0, a], \mathbf{R}^{n}\right)$, $\left\{v_{m}\right\}$ is bounded in $L^{1}\left([0, a], \mathbf{R}^{n}\right)$. Consequently, for all $m \in \mathbf{N}$ and $t \in[0, a]$ we get

$$
\begin{aligned}
\left\|\phi_{1}\left(v_{m}\right)(t)-\phi_{1}(v)(t)\right\|_{n} & \leq\left\|\int_{0}^{t} v_{m}(s) d s\right\|_{n}+\left\|\phi_{1}(v)(t)\right\|_{n} \\
& \leq\left\|\phi_{1}(v)(t)\right\|_{n}+\sup _{j \in \mathbf{N}}\left\|v_{j}\right\|_{L^{1}\left([0, a], \mathbf{R}^{n}\right)} .
\end{aligned}
$$


By the dominated convergence theorem we get

$$
\lim _{m \rightarrow+\infty} \int_{0}^{a}\left\|\phi_{1}\left(v_{m}\right)(t)-\phi_{1}(v)(t)\right\|_{n} d t=0
$$

hence $\left\{\phi_{1}\left(v_{m}\right)\right\}$ converges strongly to $\phi_{1}(v)$ in $L^{1}\left([0, a], \mathbf{R}^{n}\right)$. Of course, this implies that $\left\{\phi_{1}\left(v_{m}\right)\right\}$ also converges weakly to $\phi_{1}(v)$ in $L^{1}\left([0, a], \mathbf{R}^{n}\right)$. Consequently, using exactly the same argument as before, it is immediately seen that $\left\{\phi_{2}\left(v_{m}\right)\right\}$ also converges strongly to $\phi_{2}(v)$ in $L^{1}\left([0, a], \mathbf{R}^{n}\right)$. By using recursively the same argument as before, we get that for each fixed $j=1, \ldots, k$, the sequence $\left\{\phi_{j}\left(v_{m}\right)\right\}$ converges strongly to $\phi_{j}(v)$ in $L^{1}\left([0, a], \mathbf{R}^{n}\right)$. Of course, this implies that the whole sequence $\left\{\Phi\left(v_{m}\right)\right\}$ converges strongly to $\Phi(v)$ in $L^{1}\left([0, a], \mathbf{R}^{n k}\right)$, as desired.

(b)" Let $d$ denote the usual point-to-set distance in $\mathbf{R}^{n}$, with respect to the norm $\|\cdot\|_{n}$. Then he function

$$
\omega: t \in[a, b] \rightarrow \sup _{\xi \in \mathbf{R}^{n k}} d\left(0_{\mathbf{R}^{n}}, G(t, \xi)\right)
$$

belongs to $L^{\infty}([0, a])$ and $\|\omega\|_{L^{\infty}([a, b])} \leq M$ (as regards the measurability of $\omega$, we refer to [9]).

Therefore, all the assumptions of Theorem 2.3 are satisfied. Consequently, there exists a function $\hat{v} \in L^{\infty}\left([0, a], \mathbf{R}^{n}\right)$ and a set $K_{1} \in \mathcal{L}([0, a])$, with $m_{1}\left(K_{1}\right)=0$, such that

$$
\hat{v}(t) \in G(t, \Phi(\hat{v})(t)) \quad \text { for all } t \in[0, a] \backslash K_{1} \text {. }
$$

In particular, by (2) and by the above construction we get

$$
\hat{v}(t) \in H \subseteq \bar{B}\left(0_{\mathbf{R}^{n}}, M\right) \quad \text { for all } t \in[0, a] \backslash K_{1} .
$$

Fix $i \in\{1, \ldots, n\}$. Since $H \subseteq \mathbf{R}_{+}^{n}$, by (3) we get

$$
\hat{v}_{i}(t)>0 \text { for all } t \in[0, a] \backslash K_{1}
$$

(where $\hat{v}_{i}$ denotes the $i$ th component of $\hat{v}$ ). Then for a.e. $t \in[0, a]$ we have

$$
\phi_{1}(\hat{v})_{i}^{\prime}(t)=\hat{v}_{i}(t)>0
$$

In particular, this implies that $\phi_{1}(\hat{v})_{i}$ (which is absolutely continuous in $[0, a]$ ) is strictly increasing in $[0, a]$. By Theorem 2.4, the function $\left(\phi_{1}(\hat{v})_{i}\right)^{-1}$ is absolutely continuous in $[0, a]$. Consequently, by Theorem 2.5 , the set

$$
W_{i, 1}:=\left(\phi_{1}(\hat{v})_{i}\right)^{-1}(C)=\left\{t \in[0, a]: \phi_{1}(\hat{v})_{i}(t) \in C\right\}
$$

has null Lebesgue measure. Now, observe that

$$
\phi_{2}(\hat{v})_{i}^{\prime}(t)=\phi_{1}(\hat{v})_{i}(t) \quad \text { for all } t \in[0, a],
$$


hence, in particular,

$$
\left.\left.\phi_{2}(\hat{v})_{i}^{\prime}(t)>0 \quad \text { for all } t \in\right] 0, a\right] .
$$

Consequently, the function $\phi_{2}(\hat{v})_{i}$ is strictly increasing in $[0, a]$. Therefore, by Theorem 2.4 , the function

$$
\left(\phi_{2}(\hat{v})_{i}\right)^{-1}
$$

is absolutely continuous, hence by Theorem 2.5 it maps null-measure sets into nullmeasure sets. Therefore, if we put

$$
W_{i, 2}:=\left(\phi_{2}(\hat{v})_{i}\right)^{-1}(C)=\left\{t \in[a, b]: \phi_{2}(\hat{v})_{i}(t) \in C\right\},
$$

we get $m_{1}\left(W_{i, 2}\right)=0$. Applying recursively the same argument, if for all $j=1, \ldots, k$ we put

$$
W_{i, j}:=\left(\phi_{j}(\hat{v})_{i}\right)^{-1}(C)=\left\{t \in[0, a]: \phi_{j}(\hat{v})_{i}(t) \in C\right\}
$$

we get $m_{1}\left(W_{i, j}\right)=0$. Now, put

$$
U:=K_{0} \cup K_{1} \cup\left[\bigcup_{\substack{i=1, \ldots, n \\ j=1, \ldots, k}} W_{i, j}\right] .
$$

Of course, $m_{1}(U)=0$. Let $t^{*} \in[0, a] \backslash U$ be fixed. Since $t^{*} \notin \bigcup_{\substack{i=1, \ldots, n \\ j=1, \ldots, k}} W_{i, j}$, we immediately get

$$
\Phi(\hat{v})\left(t^{*}\right) \in\left(\mathbf{R}^{n}\right)^{k} \backslash\left[\bigcup_{i=1}^{k n} P_{k n, i}^{-1}(C)\right],
$$

hence, by (c), the function $\psi\left(t^{*}, \cdot\right)$ is continuous at $\Phi(\hat{v})\left(t^{*}\right)$. Therefore, by $(2)$ and $(\mathrm{d})^{\prime}$ we get

$$
\hat{v}\left(t^{*}\right) \in G\left(t^{*}, \Phi(\hat{v})\left(t^{*}\right)\right)=\left\{\psi\left(t^{*}, \Phi(\hat{v})\left(t^{*}\right)\right)\right\} \subseteq F\left(t^{*}, \Phi(\hat{v})\left(t^{*}\right)\right) .
$$

Hence, we have proved that

$$
\hat{v}(t) \in F(t, \Phi(\hat{v})(t)) \quad \text { for all } t \in[0, a] \backslash U \text {. }
$$

Now, take $u:=\phi_{k}(\hat{v}) \in C^{k-1}\left([a, b], \mathbf{R}^{n}\right)$. By the definition of the functions $\phi_{i}$ we get

$$
u^{(j)}(t)=\phi_{k-j}(\hat{v})(t), \quad \forall t \in[0, a], \forall j=0, \ldots, k-1,
$$

and

$$
u^{(k)}(t)=\hat{v}(t), \quad \text { for a.e. } t \in[0, a] .
$$


Therefore,

$$
\Phi(\hat{v})(t)=\left(u(t), u^{\prime}(t), \ldots, u^{(k-1)}(t)\right) \quad \text { for all } t \in[0, a],
$$

and the function $u$ satisfies the conclusion.

Step 2. We now prove the result in its full generality. Fix $y_{0} \in \mathbf{R}^{n}$ such that $y_{0}+H \subseteq$ $\mathbf{R}_{+}^{n}$. Let $\hat{F}:[0, a] \times\left(\mathbf{R}^{n}\right)^{k} \rightarrow 2^{\mathbf{R}^{n}}$ be defined by putting, for all $t \in[0, a]$ and all $\xi=$ $\left(\xi_{0}, \xi_{1}, \ldots, \xi_{k-1}\right) \in\left(\mathbf{R}^{n}\right)^{k}$,

$$
\hat{F}\left(t, \xi_{0}, \xi_{1}, \ldots, \xi_{k-1}\right)=y_{0}+F\left(t, \xi_{0}-\frac{y_{0}}{k !} t^{k}, \xi_{1}-\frac{y_{0}}{(k-1) !} t^{k-1}, \ldots, \xi_{k-2}-\frac{y_{0}}{2 !} t^{2}, \xi_{k-1}-y_{0} t\right) .
$$

Of course, for every fixed $t \in[0, a]$ the multifunction $\hat{F}(t, \cdot)$ is lower semicontinuous in $\left(\mathbf{R}^{n}\right)^{k}$. Moreover, by Lemma 2.6, $\hat{F}$ is $\mathcal{L}([0, a]) \otimes \mathcal{B}\left(\left(\mathbf{R}^{n}\right)^{k}\right)$-measurable.

By the first part of the proof, there exists $u \in W^{k, \infty}\left([0, a], \mathbf{R}^{n}\right)$ such that

$$
\begin{cases}u^{(k)}(t) \in \hat{F}\left(t, u(t), u^{\prime}(t), \ldots, u^{(k-1)}(t)\right) & \text { for a.e. } t \in[0, a], \\ u^{(i)}(0)=0_{\mathbf{R}^{n}} & \text { for all } i=0, \ldots, k-1 .\end{cases}
$$

It is immediate to check that the function $v(t)=u(t)-\frac{y_{0}}{k !} t^{k}$ satisfies the conclusion.

Proof of Theorem 1.2 Let $F^{*}:[0, a] \times\left(\mathbf{R}^{n}\right)^{k} \rightarrow 2^{X}$ be defined by setting

$$
F^{*}(t, \xi)= \begin{cases}F(t, \xi) & \text { if } \xi \in B^{k} \\ X & \text { if } \xi \notin B^{k}\end{cases}
$$

Of course, $F^{*}$ is $\mathcal{L}([0, a]) \otimes \mathcal{B}\left(\left(\mathbf{R}^{n}\right)^{k}\right)$-measurable, and for all $t \in[0, a]$ the multifunction $F^{*}(t, \cdot)$ is lower semicontinuous in $\left(\mathbf{R}^{n}\right)^{k}$. By Theorem 2.1 , there exists $u \in W^{k, \infty}\left([0, a], \mathbf{R}^{n}\right)$ such that

$$
\begin{cases}u^{(k)}(t) \in F^{*}\left(t, u(t), u^{\prime}(t), \ldots, u^{(k-1)}(t)\right) & \text { for a.e. } t \in[0, a] \\ u^{(i)}(0)=0_{\mathbf{R}^{n}} & \text { for all } i=0, \ldots, k-1 .\end{cases}
$$

It is immediate to check that $\left(u(t), u^{\prime}(t), \ldots, u^{(k-1)}(t)\right) \in B^{k}$ for all $t \in[0, a]$. Consequently, by the definition of $F^{*}$, the function $u$ solves problem (1).

Remark 3.2 It is quite easy to construct examples of applications of Theorem 1.2, where the multifunction $F$ is not jointly lower semicontinuous. For instance, one can take $a=1 / 8$, $b=1, M=8, n=k=1, F:[0, a] \times[-1,1] \rightarrow 2^{\mathbf{R}}$ defined by $F(t, x)=h(t) \cdot G(x)$, where

$$
h(t)= \begin{cases}1 & \text { if } t \in[0, a] \backslash \mathbf{Q}, \\ 2 & \text { if } t \in[0, a] \cap \mathbf{Q},\end{cases}
$$

and $G(x)=[x+2, x+3]$. It is routine matter to check that all the assumptions of Theorem 1.2 are satisfied (see $[5,6]$ ), and that the multifunction $F$ is not jointly lower semicontinuous. Therefore, Theorem 1.2 applies, while Theorem 1.1 does not. 


\section{Competing interests}

The authors declare that they have no competing interests.

\section{Authors' contributions}

All authors contributed equally and significantly in writing this article. All authors read and approved the final manuscript.

\section{Author details}

1 Department of Mathematical and Computer Sciences, Physical Sciences and Earth Sciences, University of Messina, Viale F. Stagno d'Alcontres, 31, Messina, 98166, Italy. ${ }^{2}$ Center for General Education, China Medical University, Taichung, 40402, Taiwan, ROC. ${ }^{3}$ Department of Mathematics, King Abdulaziz University, P.O. Box 80203, Jeddah, 21589, Saudi Arabia.

\section{Acknowledgements}

The second author was partially supported by the Grant MOST 103-2923-E-039-001-MY3.

\section{Received: 21 April 2016 Accepted: 4 August 2016 Published online: 23 August 2016}

\section{References}

1. Aubin, JP, Cellina, A: Differential Inclusions: Set-Valued Maps and Viability Theory. Springer, Berlin (1984)

2. Smirnov, GV: Introduction to the Theory of Differential Inclusions. Graduate Studies in Mathematics, vol. 41. Am. Math. Soc., Providence (2001)

3. Bressan, A: On differential relations with lower continuous right-hand side. An existence theorem. J. Differ. Equ. 37, 89-97 (1980)

4. Aubin, JP, Frankowska, H: Set-Valued Analysis. Birkhäuser, Boston (1990)

5. Klein, E, Thompson, AC: Theory of Correspondences. Wiley, New York (1984)

6. Himmelberg, CJ: Measurable relations. Fundam. Math. 87, 53-72 (1975)

7. Cubiotti, $P, Y a o, J C$ : Two-point problem for vector differential inclusions with discontinuous right-hand side. Appl. Anal. 93, 1811-1823 (2014)

8. Cubiotti, $P, Y a o, J C:$ On the two-point problem for implicit second-order ordinary differential equations. Bound. Value Probl. 2015, 211 (2015)

9. Naselli Ricceri, O, Ricceri, B: An existence theorem for inclusions of the type $\Psi(u)(t) \in F(t, \Phi(u)(t))$ and application to a multivalued boundary value problem. Appl. Anal. 38, 259-270 (1990)

10. Villani, A: On Lusin's condition for the inverse function. Rend. Circ. Mat. Palermo 33, 331-335 (1984)

11. Hewitt, E, Stromberg, K: Real and Abstract Analysis. Springer, Berlin (1965)

12. Anello, G: An existence theorem for an implicit integral equation with discontinuous right-hand side. J. Inequal. Appl. 2006, Article ID 71396 (2006). doi:10.1155/JIA/2006/71396 